\title{
Relevance Judgment When Browsing a Health Discussion Forum: Content Analysis of Eye Fixations
}

\author{
Wenjing Pian \\ Wee Kim Wee School of Communication \& Information \\ Nanyang Technological University, Singapore \\ wpian1@e.ntu.edu.sg \\ Christopher S.G. Khoo \\ Wee Kim Wee School of Communication \& Information \\ Nanyang Technological University, Singapore \\ chriskhoo@pmail.ntu.edu.sg \\ Yun-Ke Chang \\ Wee Kim Wee School of Communication \& Information \\ Nanyang Technological University, Singapore \\ YKChang@ntu.edu.sg
}

\begin{abstract}
Introduction. People are increasingly searching and browsing for health information on social media sites. This is a small study of the relevance criteria used by laypersons when browsing a health discussion forum under three conditions - when seeking information for their own health issue, for a friend's or relative's health issue, and with no particular issue in mind.

Method. An eye-tracker system was used to identify what text users' eyes were fixated on when browsing post surrogates and post content on a health discussion forum. Eye-fixations indicated the text segments that the user's attention was focused on when making relevance judgments.

Analysis. Content analysis was performed on the text segments with eyefixation, to identify the types of information they contain. These types of information are considered to be the direct relevance criteria used.

Results. Users seeking information for their own health issue focused on casebased information: the poster's symptom and history of disease, demographic information, and feelings about the symptom. They also focused on descriptions of disease and treatments. Participants seeking for other people's health issue focused on factual information: terminology, etiology and description of disease, and description of treatments. Participants browsing with no particular issue focused on topics of general interest such as smoking, and rare or unusual issues. Conclusion. While the relevance criteria of topicality, accuracy, currency and authority are not unimportant, they are not upper-most in the minds of users when assessing information content.
\end{abstract}




\section{INTRODUCTION}

More and more people are seeking and accessing health information on the Internet. A telephone survey of adults in the United States conducted in 2012 by PEW Internet \& American Life Project (Fox \& Duggan, 2013) found that 59\% of adults had sought health information on the Internet in the past year. $35 \%$ had accessed health information online to find out what medical condition they or someone else had. Among these "online diagnosers," $53 \%$ talked about what they found with their clinicians and $41 \%$ had their diagnosis confirmed by their clinician. Moreover, the survey found that among the online health information seekers, $16 \%$ had sought other people who shared the same concerns on the Internet.

Internet users sought various kinds of health information online. The PEW survey (Fox \& Duggan, 2013) found that among the people who looked for online health information, $53 \%$ had sought information about specific diseases or problems, $43 \%$ for particular medical treatments, $27 \%$ for weight loss and control information, $19 \%$ for food safety information and $15 \%$ for drug information. But it is not clear how people decide what health information is relevant for particular purposes (e.g., diagnosis) and in different situations, and what relevance criteria they use. Most of the previous studies on relevance judgments were in the context of information retrieval in online bibliographic or full text databases (e.g., Shawn, 1995). More recently, researchers studied relevance judgments on Web search engines (e.g., Balatsoukas \& Ruthven, 2012). They identified various types of relevance criteria used during the information search process. As social media sites become more and more popular among Internet users, it is important to know what relevance criteria people use on social media sites for relevance decisions, and whether the criteria are the same as for search engines and online databases.

There are different types of social media applications with different characteristics and information behaviour of their users. Our study focused on a health discussion forumHealthBoards.com (http://www.healthboards.com/boards/index.php). On a discussion forum, users post health issues, and view and respond to other people's posts. The posts on discussion forums are organized by topics and sub-topics in a hierarchical structure, which is different from Facebook and Instagram where people post information sequentially like a news feed, without organizing their posts. Users of a discussion forum focus on browsing by topic the posts and responses to posts, rather than checking only the responses to their own posts or the posts of specific people as in Facebook. This is different from traditional online information searching where researchers have focused on relevance judgment in the context of searching, rather than browsing. Users may exhibit different browsing and relevance judgment behaviours on social media sites. We have not come across research reports of relevance judgment in social media sites, especially in the context of browsing.

Studies of health information seeking in the past have focused on health information seeking by doctors and health professionals, for example how doctors search for medical papers in online databases (e.g., Leckie, Pettigrew \& Sylvain, 1996). Information seeking on a health discussion forum is related more to everyday life health information seeking by laypersons or consumers, rather than online database searching by health professionals. Savolainen (1995) characterized everyday life information seeking as related to the notion of "order of life" and "sense making of life," which is not related to people's jobs and work tasks. Everyday life health information seeking by the general public does not focus on obtaining professional medical knowledge. They have different reasons for carrying out health information seeking and use different kinds of relevance criteria. 
Previous studies have identified general categories of relevance criteria, such as topicality and usefulness. These abstract categories of criteria have different specific meanings in different domains and contexts. For instance, usefulness can refer to an appropriate treatment that the user can adopt to alleviate a heath condition. Few researchers have identified domain-specific detailed criteria for deciding relevance or usefulness. In specific domains such as health, more detailed criteria may be more illuminating and useful. Furthermore, identifying and coding abstract relevance criteria involves inferencing and generalization (or categorization) by the coders, and contextual and domain information is lost.

Previous studies of relevance criteria have often employed interviews, where participants were asked to recall retrospectively their experiences and talk about the reasons for their relevance decisions. This self-reporting method is based on people's recall and it is difficult to examine the details of the relevance judgment process. Another method is the questionnaire survey, using a list of relevance criteria for participants to indicate the criteria they used. This method is also based on self-reporting, and people may be influenced by the choices provided and prompted to select a criterion that seemed correct, especially when the choices are abstract and need some inferencing and generalization to select.

In this study, we recorded people's eye movements using an eye-tracker machine to identify what text they focused on, which indicate what information they used to decide relevance. This study analyzed users' eye fixations during the browsing process. Eye-fixations indicate the text that the user's attention was focused on. The information content of the text fixated on reflects the information used to make a relevance judgment, and provides evidence of the detailed relevance criteria used.

This study also distinguished between three types of information needs - users searching for their own health issue, users searching for a friend's or relative's health issue, and users who do not have any specific health issue in mind. The 2012 PEW survey mentioned earlier (Fox \& Duggan, 2013) found that among the U.S. adults seeking health information online, 39\% accessed health information for their own issues, 39\% for others, and $15 \%$ for both themselves and others. It is also possible for people to browse for health information just based on general interest and curiosity but without a particular health issue in mind. This can be considered monitoring behaviour such as when people read or scan health magazines. We investigate whether these three types of users tended to use different types of relevance criteria.

In summary, this is a study of the relevance criteria used by laypersons when browsing a health discussion forum for health information for their own health issue, other people's health issue or without a particular issue in mind. Specifically, the study investigated the types of information that users consider when they make relevance judgment, by carrying out a content analysis of the text that users' eyes fixate on. We consider these types of information to be the detailed relevance criteria. These can also be viewed as attributes of information that users use to infer a higher-level relevance category.

\section{LITERATURE REVIEW}

Many researchers have developed typologies of relevance criteria, and identified the relevance criteria used in different contexts. Cool, Belkin, Frieder and Kantor (1993) investigated how people make relevance judgment on documents in the context of an information retrieval system, and derived a list of relevance criteria consisting of six big categories (i.e. topic, content/information, format, presentation, values and oneself), and 50 
detailed criteria items, such as define the topic, part of topic, writing style and understandability. Some of the criteria are more useful for assessing the relevance of documents (i.e. containers of information) than for assessing actual information content, such as the following:

- Topic, in this study is mainly the disease or health condition, which is defined by the section of the discussion forum. It is not a criterion that the user has to consciously assess and keep in attention, as most of the postings in the section are on the particular health condition. Relevant topic is the default attribute that is assumed unless there is clear evidence to contrary, in which case the user's attention is alerted. More detailed topical considerations, such as whether a document provides a deep or superficial treatment of a topic and whether it directly focuses on the topic, are probably not important as users are looking for any information that is useful or interesting.

- Format of the document is fixed by the type of social media application (i.e. discussion forum) which determines the format.

- Presentation (e.g., organization, writing style and whether technical language is used) may influence relevance judgment, but the effect is subtle and it is difficult to determine its importance in the context of social media as most of the posts are written in layperson conversational style.

The following three criteria from Cool, Belkin and Kantor (1993) are more important to this study:

- Content/information refers to factual information about health conditions and treatments. Sub-categories include basic concepts, definitions, connections, tips, guidelines and level of detail.

- Oneself (relationship with the user's situation) is the focus of this study. We attempt to identify the types of information that are used to assess the relevance to the user's situation.

- Values are dimensions that modify other criteria. The values of interest and entertainment are important for users who are browsing with no particular health issue in mind. The other dimensions of specificity (specific/general), precision (precise/vague), bias and authority are also potentially important in assessing the relevance of information content.

Schamber (1991) conducted an interview of 30 people who used weather information in their jobs about how they judged the relevance of information from newspapers, TV and radio broadcasting. She derived a list of 21 categories of relevance criteria from the interview data that she grouped into 10 groups: accuracy, currency, specificity, geographical proximity, reliability, accessibility, verifiability, clarity, dynamism (changing over time) and presentational quality. Among these criteria, specificity is most applicable to this study.

Saracevic (2007) reviewed the literature on previous user studies of relevance criteria and relevance judgment, and grouped the relevance criteria into seven big categories: content, object, validity, situation match, cognitive match, affective match and belief match. Each category included several detailed relevance criteria items. Situation match mentioned in this list refers to appropriateness to situation or tasks and usability, which are important in the context of health information seeking. When people seek health information for their own health issue, they will attempt to match the information to their personal characteristics or health issue. Affective match includes fun and interest, which may be important in the situation when people browse health information with no particular issue in mind.

Crystal and Greenberg (2006) conducted a study to identify user relevance criteria for health information during Web searching. They found that the categories of topic, scope, data, Web characteristics, affiliation, authority, influence and methodology were key criteria user 
used in relevance judgment. They suggested that more research into metadata related to users (i.e. user-centeredness and fit to user's situation and tasks) will provide more insights into relevance judgment.

To summarize, previous studies have identified categories of relevance criteria at a fairly abstract and general level that are focused on document relevance rather than information relevance, and to academic contexts of finding research articles for research report writing or developing research studies. Criteria such as situation match or affective match (i.e. emotions) are likely to be more important in health information browsing, and this study sought to shed more light on these criteria and identify more detailed criteria.

\section{RESEARCH METHOD}

We adopted the model of information seeking on social media by Zhang (2012) as the overall model. Her model specifies the information seeking stages of information needs, identifying information source, examining and evaluating information, interpreting (using) information, and information need met. Among these stages, the examining and evaluating stage is related to the interaction between user and the system, which is the focus of this study. We subdivide examining and evaluating into the following more detailed stages:

- User searching (by submitting keywords in a search box) or browsing (the classification directory) to retrieve and display post surrogates matching the query or directory category.

- Scanning a summary result screen displaying a list of retrieved post surrogates (usually the title of the posts). The purpose of scanning is to select a post surrogate to click on to display the detailed post screen with the content of the post, together with other users' responses to the post.

- Reading the detailed post screen.

This study focused not on the searching and directory browsing stage but on the scanning and reading stages. We subdivide these two stages into two types of behaviour:

- Skimming quickly over the text or the items on the screen

- Examining the text or items more closely.

During skimming, the user's eyes go over the text or surrogates quickly, seeking text or surrogate titles of potential interest to focus the user's attention on. It is likely that salient words in the text attract the user's attention during skimming. We consider this an unconscious kind of relevance judgment involving a fast cognitive process that is not available to conscious introspection. Previous authors (e.g., Zhang, 2012) have also assumed that this attraction of the user's attention or focus during skimming is keyword-based. Skimming was not included in the scope of this study, as our interest was in the deliberate relevance judgment when the user's attention is engaged. In this study, we focused on the examining stage of users' interaction with the discussion forum:

- examining a post surrogate to estimate the likelihood that the post contains relevant information, and

- examining a text segment (e.g., sentence or paragraph) of a post content to decide whether the information expressed is relevant or potentially relevant to a use context.

Table 1 shows our model of user interaction with a discussion forum, focusing on the type of relevance judgment at each stage of the interaction.

Fourteen graduate students were recruited for this study. They were asked to search for health information relevant either to their own health issue, or a friend's or relative's health issue. If they could not think of any health issue that they or a friend/relative had, then they were asked to browse the discussion forum with no particular health issue in mind. Six 
Table 1. Model of user interaction with a discussion forum, focusing on relevance judgment

\begin{tabular}{ll}
\hline User information behaviour & Decision making (i.e. relevance judgment) \\
\hline Stage 1. Searching or Browsing & $\begin{array}{l}\text { System retrieves and displays surrogates } \\
\text { matching search query or selected directory } \\
\text { category (relevance judgment by machine) }\end{array}$ \\
\hline
\end{tabular}

Stage 2. Scanning (the surrogates in the summary result screen)
a. Skimming
Select surrogates for attention/focus
(unconscious relevance judgment)
b. Examining
Select surrogates to retrieve the associated (linked) document (conscious relevance judgment based on an estimation of the likelihood that the document contains relevant/useful information)

\section{Stage 3. Reading}

a. Skimming (through post content quickly)

Select text for attention/focus (unconscious relevance judgment)

b. Examining and absorbing the information in the text/content of Deciding whether the information is relevant or likely to be useful in a use context document (conscious relevance judgment)

participants chose to search for their own health issue, two decided to search for a friend's or relative's issue, and four searched with no particular issue. The last two participants were asked to search for a friend's or relative's issue.

The participants' eye movements on the screen were recorded by an eye-tracker system. The eye-tracker system used in the study was the Tobii X60 series. The device comprised a 17 -inch screen with the eye tracker embedded in it. The eye-tracker used a $60-\mathrm{Hz}$ sampling rate, had a 0.5 degree gaze point accuracy, and permitted free head motion. The system was calibrated for each subject before the start of the formal user session.

Figure 1 shows a screen capture of a detailed post screen with an aggregated representation of a participant's eye fixations. The green circles on the screen represent the participant's eye fixations on particular pieces of text. The size of the circles represents the duration of each fixation.

Content analysis was carried out of the post surrogates in the summary result screen that the participants examined (indicated by eye-fixations), and the text segments in the detailed post content that the participant examined. The surrogate titles and text segments that the participant examined were coded into types of information that they contained. Postexperiment interviews were carried out to obtain more insights into the relevance decisionmaking process and relevance criteria used. 


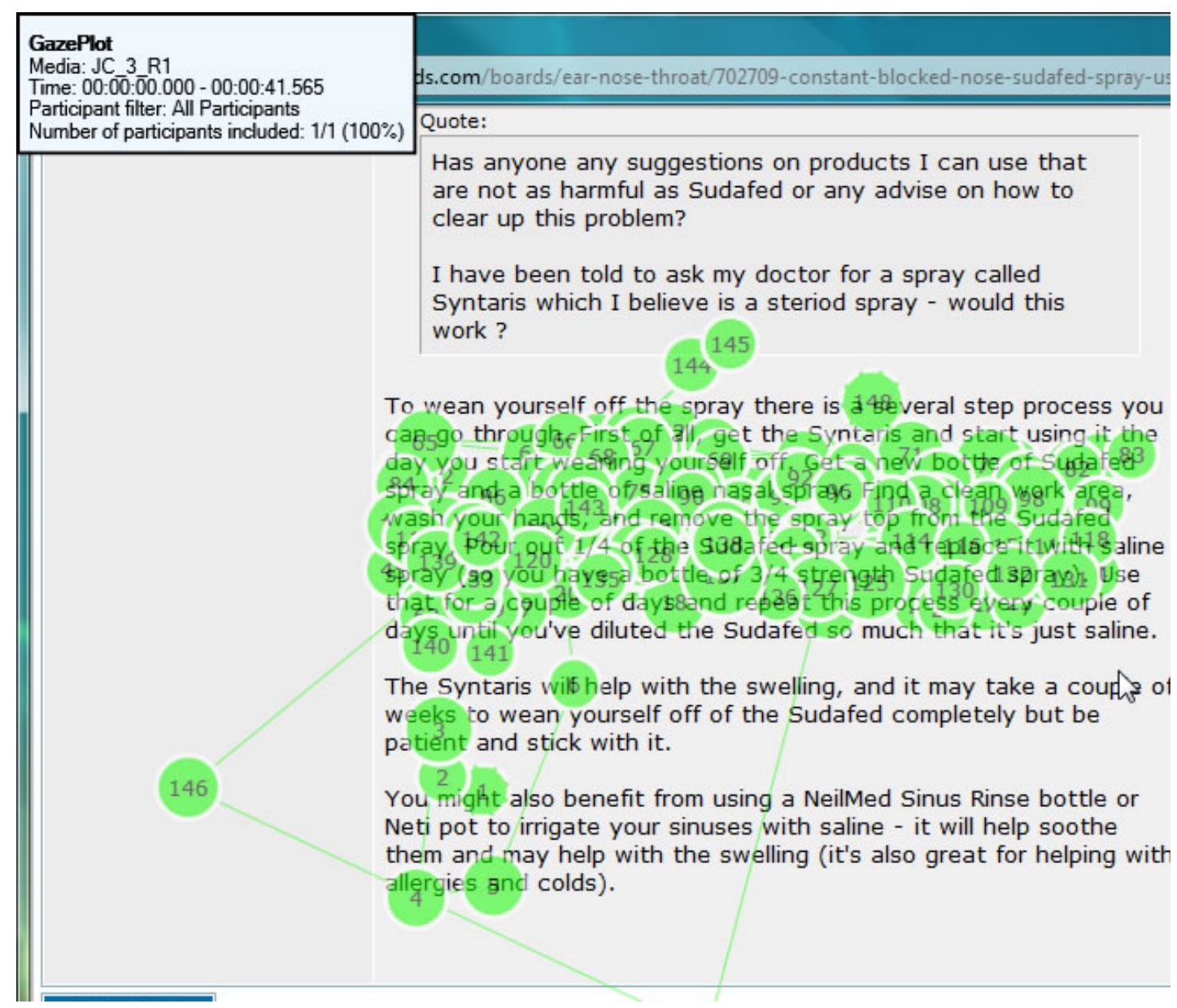

\section{Figure 1. Screen capture of a detailed post screen with an aggregated representation of a user's eye fixations}

The coding scheme of information types was derived from the following sources:

- previous literature on health information seeking on the Internet (Eysenbach, Powell, Kuss, \& Sa, 2002; Frost \& Massagli, 2008)

- external medical terminologies (Beale \& Heard, 2007; Blobel, 2011), and healthcare reference manuals (Bodenheimer, Lorig, Holman, \& Grumbach, 2002; Lorig et al., 2001; Lyder, 2003)

- relevance criteria mentioned by the participants in the post-experiment interviews

- open coding of the post surrogates and post content that users examined or focused on.

From the post-experiment interviews, we noted that users with the three types of information needs tended to use different relevance criteria:

1. Users searching for own health issue tended to use case-based criteria, i.e. attributes of the poster, the disease condition or the context that they attempted to match with their own situation.

2. Users searching for other's health issue tended to use factual knowledge-based criteria, i.e. factual knowledge about the disease or treatment that they could learn from the post

3. Users searching with no particular issue tended to use general awareness-based criteria, i.e. they identified issues that were topical or of general interest that were independent of particular diseases. 
Table 2. Coding scheme for types of information

\begin{tabular}{|c|c|c|}
\hline $\begin{array}{l}\text { Case-based relevance } \\
\text { criteria }\end{array}$ & $\begin{array}{l}\text { Factual knowledge-based } \\
\text { criteria }\end{array}$ & $\begin{array}{l}\text { General awareness-based } \\
\text { criteria }\end{array}$ \\
\hline $\begin{array}{l}\text { A. Patient demographic } \\
\text { A1. Age \& gender } \\
\text { A2. Job } \\
\text { A3. Nationality }\end{array}$ & $\begin{array}{l}\text { E. Etiology } \\
\text { E1. Cause of disease } \\
\text { E2. Description of disease } \\
\text { E3. Terminology of disease }\end{array}$ & $\begin{array}{l}\text { H. General health issue } \\
\text { H1. Common health issue } \\
\text { H2. Pollution } \\
\text { H3. Smoking } \\
\text { H4. Hot topic of general interest }\end{array}$ \\
\hline $\begin{array}{l}\text { B. Patient's symptom } \\
\text { B1. Symptom description } \\
\text { B2. Subjective feeling of having } \\
\text { the symptom } \\
\text { B3. Poster's experience of } \\
\text { symptom } \\
\text { B4. Personal history of disease }\end{array}$ & $\begin{array}{l}\text { F. Drug information } \\
\text { F1. Efficacy } \\
\text { F2. Indications } \\
\text { F3. Contra-indications } \\
\text { F4. Interaction with other drugs } \\
\text { F5. Pharmacology }\end{array}$ & $\begin{array}{l}\text { I. Curiosity-based criterion } \\
\text { I1. Rare issue } \\
\text { I2. Interesting story } \\
\text { I3. Famous people } \\
\text { I4. Counter-intuitive } \\
\text { information }\end{array}$ \\
\hline $\begin{array}{l}\text { C. Attitude \& emotion } \\
\text { C1. Attitude to the problem } \\
\text { C2. Emotional status of } \\
\text { knowing the problem } \\
\text { C3. Other's attitude \& } \\
\text { emotional support }\end{array}$ & $\begin{array}{l}\text { G. Procedure (formal) } \\
\text { G1. Type of procedure (physical, } \\
\text { chemical, biomedical, etc.) } \\
\text { G2. Description of procedure }\end{array}$ & \\
\hline $\begin{array}{l}\text { D. Patient's experience of } \\
\text { drug/treatment } \\
\text { D1. Perceived side effect } \\
\text { D2. Interaction with another } \\
\text { health problem (experienced by } \\
\text { patient) } \\
\text { D3. Dosage used } \\
\text { D4. Description of procedure } \\
\text { used } \\
\text { D5. Caution or reminder (based } \\
\text { on patient's experience) }\end{array}$ & & \\
\hline
\end{tabular}

Accordingly, we divided the information types in the coding scheme into these three broad categories, as shown in Table 2.

\section{RESULTS}

\section{Scanning Stage-Post Surrogates Focused on}

We counted the post surrogates with eye fixations for each study participant, and the post surrogates they selected (clicked on) to view the detailed posts. The results are summarized in Table3.

Participants seeking for their own health issue $(\mathrm{N}=6)$ had focused on 38 post surrogates on average (ranging from 33 to 40). Participants seeking for other's health issue $(\mathrm{N}=4)$ had 
Table 3. Average number of post surrogates with fixations and proportion selected

\begin{tabular}{lccc}
\hline & $\begin{array}{c}\text { For own issue } \\
(\mathrm{N}=6)\end{array}$ & $\begin{array}{c}\text { For others' } \\
\text { issue }(\mathrm{N}=4)\end{array}$ & $\begin{array}{c}\text { No particular } \\
\text { issue }(\mathrm{N}=4)\end{array}$ \\
\hline $\begin{array}{l}\text { Post surrogates with fixations } \\
\text { (average no. per participant) }\end{array}$ & $38(\mathrm{SD}=3.9)$ & $40(\mathrm{SD}=6.9)$ & $23(\mathrm{SD}=6.6)$ \\
& $25.7 \%(58 / 226)$ & $18.6 \%(30 / 161)$ & $27.4 \%(25 / 91)$ \\
$\begin{array}{l}\text { Overall percentage of fixated } \\
\text { surrogates selected }\end{array}$ & & & \\
\hline
\end{tabular}

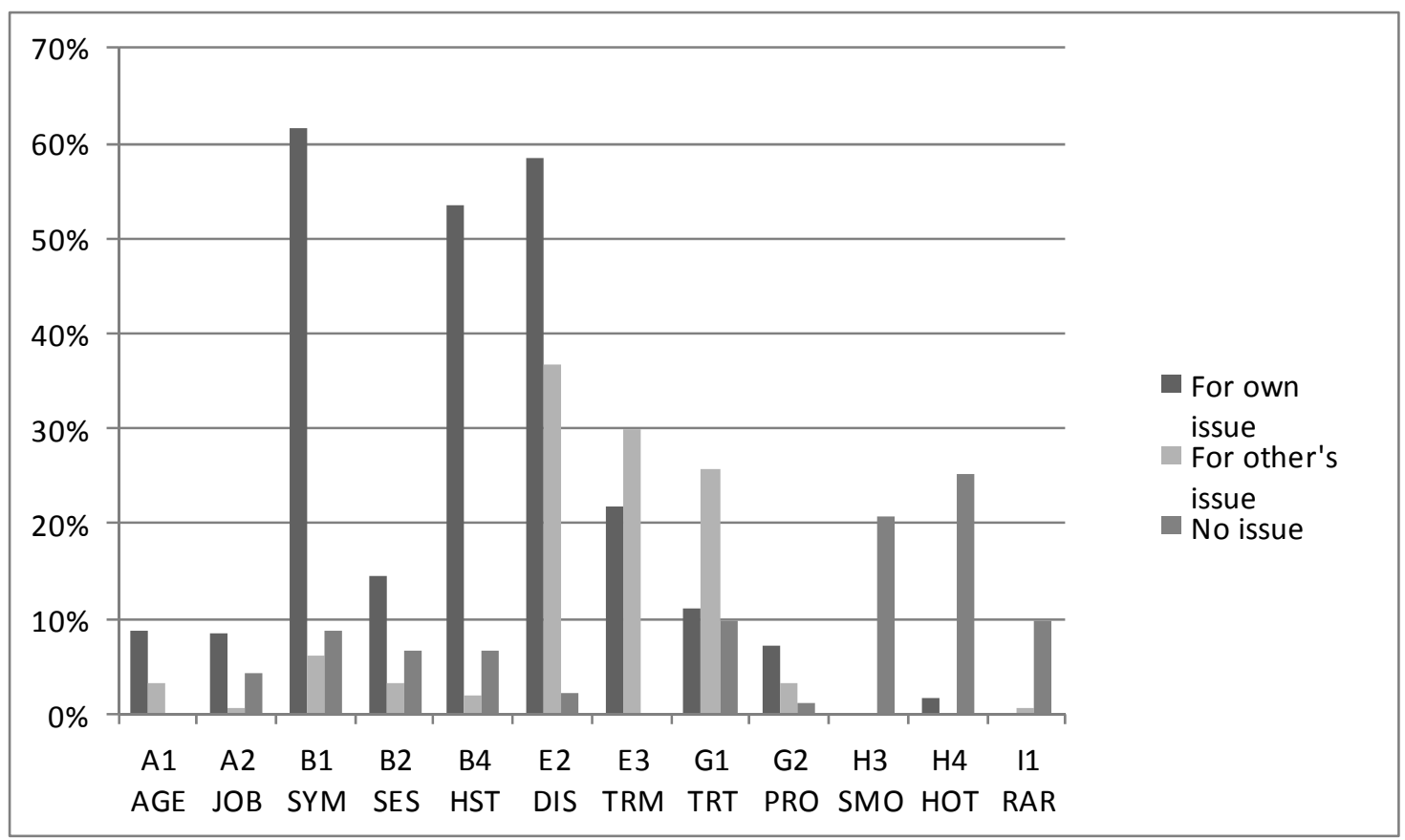

Note: Types of information occurring less than $5 \%$ of the time are left out

Figure 2. Percentage of post surrogates with eye fixation containing each type of information

focused on 40 post surrogates on average ( 32 to 48 ), which is comparable to participants searching for own health issue. In contrast, people browsing with no particular issue had focused on only 23 post surrogates on average (15 to 30). Table 3 indicates that the standard deviation for the average number of fixated surrogates was smallest for the participants seeking for their own issue, suggesting that this group of participants exhibited more consistent behaviour.

The post surrogates contained different types of health information as listed in Table 4 . Figure 2 shows the proportion of fixated post surrogates containing each type of information, for the three groups of participants. The types of information with percentages less than $5 \%$ for all three groups of participants are left out of the bar graph. Figure 2 indicates that the three groups of participants focused on post surrogates containing different types of information. 
Table 4. Percentages of types of health information in skimmed versus fixated surrogates

\begin{tabular}{lcc}
\hline Type of health information & $\begin{array}{c}\text { \% in surrogates } \\
\text { skimmed over }\end{array}$ & $\begin{array}{c}\text { \% in surrogates } \\
\text { fixated on }\end{array}$ \\
\hline For own heath issue & & \\
B1. Symptom description & 24.2 & 61.5 \\
B4. History of disease & 17.2 & 53 \\
E2. Description of disease & 30 & 58 \\
For other's health issue & & \\
E3. Terminology of disease & 11.3 & 29.9 \\
E2. Description of disease & 14.2 & 36.8 \\
G1. Type of procedure & 7.1 & 25.8 \\
G2. Description of procedure & 0.5 & 3.1 \\
& & \\
No particular issue & & 25.2 \\
H4. Hot topics & 13.1 & 20.1 \\
H3. Smoking & 8.9 & 9.9 \\
I1. Rare issue & 2.4 & \\
\hline
\end{tabular}

For participants seeking for their own health issue, the following are the most common types of information focused on:

- Description of poster's symptoms (B1: 61.5\%),

- Description of disease (E2: $58 \%$ ) and

- Personal history of disease (B4: 53\%).

In comparison, for the participants seeking for other's health issue, the most common types of information focused on were:

- Description of disease (E2: $36.7 \%$ )

- Terminology of disease (E3: 29.3\%) and

- Type of procedure (G1: 25.8\%).

For the participants browsing with no particular issue, the most common types of information focused on were:

- Hot health topic (H4: 25.3\%) (e.g., sexual issue, weight control),

- Smoking issue (H3: 20.9\%) and

- Rare health issue (I1: 9.2\%).

Comparing the percentages across the three groups of participants in Figure 2, it was found that each group of participants focused on some types of health information relatively more than other groups. We identified the percentages that were substantially higher than for the other groups (at least $50 \%$ higher):

- For participants seeking for own health issue: age (A1), job (A2), symptom description (B1), subjective feeling of having the symptom (B2), personal history of disease (B4), description of disease (E2) and description of procedure (G2).

- For participants seeking for others: terminology of disease (E3) and type of treatment (G1). 
- For participants with no particular issue: hot topics of general interest (H4), smoking (H3) and rare issue (I1).

For these types of information, we compared the percentages in the fixated post surrogates with the post surrogates that were skimmed, which can be considered the baseline percentages. This was to confirm that the percentages for these types of information in the fixated post surrogates were higher than the distribution among the post surrogates in general. We randomly selected three scanning sessions for each participant and coded the post surrogates without any eye fixations.

Table 4 shows the percentages of particular types of information for fixated surrogates in comparison to surrogates without fixations. It is clear that the percentages for the fixated surrogates are much higher than for the surrogates without fixations, indicating that the participants' eye-fixations were not random but were biased to these types of information, and the bias depended on the type of information need.

\section{Scanning Stage—Post Surrogates Selected}

We analyzed the post surrogates that the participants actually selected (clicked on) to display the post content. It was found that for participants seeking for own health issue, a high proportion of selected post surrogates contained the following types of information:

- Description of poster's symptom (B1: 88\%),

- Personal history of disease (B4: 75\%), and

- Description of disease (E2: 72\%).

For those seeking for other people's issue:

- Terminology description (E3: 77\%),

- Description of disease (E2: 72\%) and

- Description of procedure (G2: $16.7 \%)$.

For those with no particular issue:

- Hot health topic (H4: 68\%)

- Smoking issue (H3: 56\%) and

- Rare health issue (I1: 28\%).

The types of information listed above are the same as those found for fixated post surrogates (described in the previous section), except in much higher proportions.

It may be more revealing to analyze the data in terms of the percentage of the fixated post surrogate that was selected. Table 5 lists for each type of information the percentage of fixated posts containing the information that were actually selected (i.e. clicked on). Table 3 has earlier indicated that participants seeking for their own issue selected $25.7 \%$ of the surrogates they focused on, to display the post content. Thus, in Table 5 column 2, percentages above $26 \%$ are above the baseline overall percentage of fixated post surrogates selected.

Participants seeking for others selected only $18.6 \%$ of the surrogates they focused on. This is the baseline percentage for column 3 of Table 5. Participants browsing with no particular issue selected $27.4 \%$ of the surrogates focused on. Though this percentage is higher than for the participants searching for others, the actual number of surrogates selected is lower: 25 surrogates, compared to the 30 selected by the four participants searching for others.

The types of information that had substantially higher percentage selected than the baseline are:

- For own health issue: age (A1), job status (A2), nationality (A3), personal symptom (B1), subjective feeling of having the symptom (B2), personal history of disease (B4), dosage of 
Table 5. Percentage of fixated post surrogates containing each type of information that were actually selected for detailed display

\begin{tabular}{llll}
\hline Type of health information & $\begin{array}{l}\text { For own } \\
\text { health issue }\end{array}$ & $\begin{array}{l}\text { For other's } \\
\text { health issue }\end{array}$ & $\begin{array}{l}\text { No particular } \\
\text { issue }\end{array}$ \\
\hline A1. Age & $55 \%(11 / 20)$ & - & - \\
A2. Job & $42 \%(8 / 19)$ & - & - \\
A3. Nationality & - & - & - \\
B1. Symptom description & $37 \%(51 / 139)$ & $30 \%(3 / 10)$ & - \\
B2. Subjective feeling of having the & & & - \\
symptom & $47 \%(14 / 33)$ & - & - \\
B4. Personal history of disease & $37 \%(44 / 121)$ & - & - \\
E2. Description of disease & $37 \%(42 / 132)$ & $82 \%(22 / 27)$ & - \\
E3. Terminology of disease & $37 \%(42 / 132)$ & $77 \%(23 / 32)$ & - \\
G1. Type of treatment & $40 \%(10 / 25)$ & $64 \%(12 / 19)$ & - \\
G2. Description of procedure & $50 \%(8 / 16)$ & - & - \\
H3. Smoking & - & - & $74 \%(14 / 19)$ \\
H4. Hot topic of general interest & - & - & $74 \%(17 / 23)$ \\
I1. Rare issue & - & - & $78 \%(7 / 9)$ \\
\hline Overall percentage & $\mathbf{2 6 \%}$ & $\mathbf{1 9 \%}$ & $\mathbf{2 7 \%}$ \\
\hline
\end{tabular}

* Categories with fewer than 10 surrogates for the fixated surrogates were left out

drug (D3), drug indication (F2), description of disease (E2), disease terminology (E3), type of procedure/treatment (G1) and description of procedure/treatment (G2).

- For other's health issue: personal symptom (B1), cause of disease (E1), disease description (E2), terminology of disease (E3), type of procedure/treatment (G1) and description of procedure/treatment (G2).

- For no particular issue: cause of disease (E1), drug efficacy (F1), smoking issue (H3), hot public topic (H4), rare health issue (I1) and interesting stories (I2).

Based on above criterion, it can be concluded that for participants seeking information for their own health issue, symptom (B1, B2, B4) and treatment information (E2, E3, G1, G2) played a big role in their determining the post surrogate relevance. For participants seeking for other's issue, factual information about treatment and disease (E2, E3, G1) played a big role. In contrast, for participants browsing with no particular issue, general interest (H3, H4) and curiosity (I1) played a big role. The three groups of participants used different kinds of relevance criteria in their relevance decisions.

\section{Reading Stage—Post Content Judged Relevant}

In this stage, participants read the selected post content (and responses from other users), and judged the relevance of the content they focused on (text segment with fixation). Table 6 shows summary statistics of selected posts and posts judged relevant. Participants seeking information for own health issue had the highest percentage of posts judged relevant. However, participants with no particular health issue read the most posts but had the lowest percentage of relevant posts. 
Table 6. Percentage of selected posts judged relevant

\begin{tabular}{lccc}
\hline $\begin{array}{l}\text { Group of } \\
\text { participants }\end{array}$ & $\begin{array}{c}\text { No. of selected } \\
\text { posts }\end{array}$ & $\begin{array}{c}\text { No. of posts judged } \\
\text { relevant }\end{array}$ & $\begin{array}{c}\text { Percentage of } \\
\text { relevant post }\end{array}$ \\
\hline $\begin{array}{l}\text { For their own issue } \\
(\mathrm{N}=6)\end{array}$ & 58 & 48 & $82.8 \%$ \\
$\begin{array}{l}\text { For other's issue } \\
(\mathrm{N}=4)\end{array}$ & 30 & 17 & $56.7 \%$ \\
$\begin{array}{l}\text { No particular issue } \\
(\mathrm{N}=4)\end{array}$ & 25 & 7 & $28 \%$ \\
\hline
\end{tabular}

Table 7. Percentage of selected posts containing each type of information that were judged relevant

\begin{tabular}{lc}
\hline Type of health information & $\begin{array}{c}\text { \% of selected } \\
\text { posts judged } \\
\text { relevant }\end{array}$ \\
\hline For own health issue (baseline = 82.8\%) & \\
Symptom description (B1) & $82.4 \%$ \\
Personal history of disease (B4) & $67.7 \%$ \\
Description of procedure/treatment (G2) & $74.4 \%$ \\
& \\
For other's health issue (baseline $=\mathbf{5 6 . 7 \%})$ & \\
Cause of disease (E1) & $62.0 \%$ \\
Description of disease (E2) & $57.7 \%$ \\
Description of procedure/treatment (G2) & $57.7 \%$ \\
& \\
No particular issue (baseline $=\mathbf{2 8 \% )}$ & \\
Air/water pollution (H2) & $66.7 \%$ \\
Smoking issue (H3) & $25.0 \%$ \\
Rare issue (I1) & $25.0 \%$ \\
\hline
\end{tabular}

The analysis in this section focuses on the text segments (sentences) that the participants focused on (indicated by eye-fixation) in the post content. A post was considered to contain a particular type of information if the sentences that the participant focused on in the post contained the type of information. In other words, sentences in the post that the participant skimmed through or ignored were not considered.

We calculated the percentage of the selected posts containing each type of health information that were judged relevant. Table 7 lists the types of information with a high percentage of selected posts judged relevant.

The types of health information with the highest percentage of posts judged relevant were largely similar to the information types that were important in the scanning stage. However, as the post content contains much more detailed information than the post surrogates (which comprise mainly post titles), the results indicated more emphasis on 
detailed information. For participants seeking for own health issue, there was more emphasis on the description of the procedure/treatment (G2), rather than just the type of procedure (G1). For participants seeking for other people's issue, there was more focus on the cause of the disease (E1), in addition to the description of the disease (E2), as well as the description of the procedure/treatment (G2). For participants with no particular issue, topical issues of general interest remained important as well as curious and unusual issues. The topical issue of air/water pollution gained prominence in this phase. The prominence of pollution topics was because Singapore was suffering from haze pollution caused by forest fires in neighbouring Indonesia. This result indicates that local health issues do influence users' relevance judgments during information browsing.

\section{CONCLUSION AND DISCUSSION}

The study found that participants seeking information for their own health issue and other people's health issue had about the same number of post surrogates with eye-fixations. However, participants searching for their own issue had a smaller standard deviation indicating more consistent behaviour. They also selected more surrogates to view the detailed content. Participants with no issue had much fewer eye-fixations, but about the same number of surrogates selected as participants searching for others. These behaviours can partly be explained by the amount of effort each group of participants were willing to invest in the information seeking process: users searching for their own health issue were willing to put in more effort. In addition, users with no particular issue in mind exerted more effort in skimming for interesting information than in attentive examination.

For participants seeking information for their own health issue, when scanning post surrogates, they focused mainly on the poster's symptoms and history of disease, and terminology and description of disease. To a smaller extent they also focused on the poster's age, occupation, feelings about the symptom, and type and description of treatment. When reading the detailed post content, they focused on the same kinds of information with more emphasis on the details of the treatments. Data from post-experiment interviews indicate that this group of participants generally engaged in case-based reasoning to match post surrogates and post content with their own situation. This is supported by the results of our analysis of eye-fixation data, which indicate that the participants paid more attention to the posters' demographic information, health condition and subjective experience.

Participants seeking information for other people's issue focused on the terminology and description of the disease, and type of treatment when scanning post surrogates. When reading post content, more focus was placed on the etiology and cause of disease, and description of the treatment. This is consistent with data from the post-experiment interviews, which indicate that this group of participants were looking for factual information/knowledge of the disease and treatments.

Participants browsing with no particular issue focused on topics of general interest such as smoking, pollution, sexual issues and weight loss, and rare or unusual issues.

The types of information that users focus on during browsing can be considered to be more detailed relevance criteria within the broader categories of content/information, oneself and values in the framework of Cool, Belkin and Kantor (1993). This does not mean that the relevance criteria of topicality, accuracy, currency, authority, reliability, presentation, etc. identified by various researchers are not important. They are just not upper-most in the minds of users when deciding on the relevance of information content. Perhaps the users were adopting some assumed default values for these criteria. These relevance criteria might be 
alerted to the user's attention when the assumptions are violated in the post content. These criteria might also become an important consideration later when users decide to actually use the information or adopt a recommendation in practice.

Data from the post-experiment interviews indicate that participants did pay some attention to information about the poster and who the poster was. This suggests that the authority of the post writer was considered during the relevance judgment, but perhaps the experimental environment wasn't conducive for the participants to explore who the posters were in more detail.

\section{REFERENCES}

Balatsoukas, P., \& Ruthven, I. (2012). An eye-tracking approach to the analysis of relevance judgments on the Web: The case of Google search engine. Journal of the American Society for Information Science and Technology, 63(9), 1728-1746.

Beale, T., \& Heard, S. (2007). An ontology-based model of clinical information. Studies in Health Technology and Informatics, 129(1), 760-764.

Blobel, B. (2011). Ontology driven health information systems architectures enable pHealth for empowered patients. International Journal of Medical Informatics, 80(2), e17-e25.

Bodenheimer, T., Lorig, K., Holman, H., \& Grumbach, K. (2002). Patient self-management of chronic disease in primary care. JAMA: The Journal of the American Medical Association, 288(19), 2469-2475.

Cool, C., Belkin, N., Frieder, O., \& Kantor, P. (1993). Characteristics of text affecting relevance judgments. In M. E. Williams (Ed.), Proceedings of the 14th National Online Meeting (pp. 77-84). Medford, NJ: Learned Information, Inc.

Eysenbach, G., Powell, J., Kuss, O., \& Sa, E. (2002). Empirical studies assessing the quality of health information for consumers on the world wide web: A systematic review. JAMA: the Journal of the American Medical Association, 287(20), 2691-2700.

Fox, S., \& Duggan, M. (2013). Health online 2013. Washington, D.C.: Pew Research Center's Internet \& American Life Project. Retrieved from http://www.pewinternet.org/files/oldmedia/Files/Reports/PIP_HealthOnline.pdf

Frost, J. H., \& Massagli, M.P. (2008). Social uses of personal health information within PatientsLikeMe, an online patient community: What can happen when patients have access to one another's data. Journal of Medical Internet Research, 10(3), e15. Retrieved from http://www.jmir.org/2008/3/e15/

Leckie, G. J., Pettigrew, K.E., \& Sylvain, C. (1996). Modeling the information seeking of professionals: A general model derived from research on engineers, health care professionals, and lawyers. The Library Quarterly, 66(2), 161-193.

Lorig, K.R., Ritter, P., Stewart, A.L., Sobel, D.S., Brown Jr, B.W., Bandura, A., ... Holman, H.R. (2001). Chronic disease self-management program: 2-year health status and health care utilization outcomes. Medical Care, 39(11), 1217-1223.

Lyder, C. H. (2003). Pressure ulcer prevention and management. JAMA: The Journal of the American Medical Association, 289(2), 223-226.

Saracevic, T. (2007). Relevance: A review of the literature and a framework for thinking on the notion in information science. Part III: Behavior and effects of relevance. Journal of the American Society for Information Science and Technology, 58(13), 2126-2144.

Schamber, L. (1991). Users' criteria for evaluation in a multimedia environment. Proceedings of the ASIS Annual Meeting, 28, 126-133. 
Libres volume 24, issue 2, pages 132-147

Zhang, Y. (2012). Consumer health information searching process in real life settings.

Proceedings of the American Society for Information Science and Technology, 49(1), 110. 